\title{
Final results of the DAPS (Denosumab Adherence Preference Satisfaction) study: a 24-month, randomized, crossover comparison with alendronate in postmenopausal women
}

\author{
N. Freemantle $\cdot$ S. Satram-Hoang $\cdot$ E.-T. Tang $\cdot$ \\ P. Kaur $\cdot$ D. Macarios $\cdot$ S. Siddhanti $\cdot$ J. Borenstein . \\ D. L. Kendler • on behalf of the DAPS Investigators
}

Received: 22 April 2011 / Accepted: 22 August 2011 /Published online: 17 September 2011

(C) The Author(s) 2011. This article is published with open access at Springerlink.com

\begin{abstract}
Summary The final analysis of this 2-year, randomized, crossover study showed that postmenopausal women with osteoporosis were more adherent, compliant, and persistent with subcutaneous denosumab injections every 6 months than with once-weekly alendronate tablets. After receiving both treatments, women reported greater satisfaction with injectable denosumab and preferred it over oral alendronate.
\end{abstract}

After the study, Dr. Freemantle's affiliation changed to University College London, UK.

Electronic supplementary material The online version of this article (doi:10.1007/s00198-011-1780-1) contains supplementary material, which is available to authorized users.

\author{
N. Freemantle \\ University of Birmingham, \\ Birmingham, UK \\ S. Satram-Hoang $\cdot$ E.-T. Tang $\cdot$ P. Kaur $\cdot$ D. Macarios $\cdot$ \\ S. Siddhanti \\ Amgen Inc., \\ Thousand Oaks, CA, USA \\ J. Borenstein \\ Cedars-Sinai Health System, \\ Beverly Hills, CA, USA \\ D. L. Kendler \\ University of British Columbia, \\ Vancouver, BC, Canada \\ N. Freemantle $(\bowtie)$ \\ Department of Primary Care and Population Health, \\ Upper Third Floor, UCL Medical School (Royal Free Campus), \\ Rowland Hill Street, \\ London NW3 2PF, UK \\ e-mail: nicholas.freemantle@ucl.ac.uk \\ P. Kaur \\ David Geffen School of Medicine, \\ UCLA Los Angeles, CA, USA
}

Introduction Osteoporosis patients who are non-compliant or non-persistent with therapy may have suboptimal clinical outcomes. This 2-year, randomized, open-label, crossover study compared treatment adherence between subcutaneous denosumab, $60 \mathrm{mg}$ every 6 months, and oral alendronate, $70 \mathrm{mg}$ once weekly.

Methods Postmenopausal women at 25 centers in the USA and Canada with bone mineral density T-scores -4.0 to -2.0 and no prior bisphosphonate use received alendronate then denosumab, or denosumab then alendronate, over successive 12-month periods. Adherence required both compliance (denosumab injections 6 months apart or $\geq 80 \%$ of alendronate tablets) and persistence (both denosumab injections or $\geq 2$ alendronate doses in the last month and completion of the treatment period).

Results Of the 250 women enrolled (124 alendronate, 126 denosumab), 221 entered the second year (106 denosumab, 115 alendronate). Denosumab was associated with less nonadherence than alendronate (first year, $11.9 \%$ vs $23.4 \%$; second year, $7.5 \%$ vs $36.5 \%$ ). Risk ratios for non-adherence, non-compliance, and non-persistence favored denosumab in both years $(p<0.05)$. Of 198 subjects expressing treatment preference, $183(92.4 \%)$ preferred the injections over the oral therapy. BMD improved further when subjects received denosumab after alendronate and remained stable when they received alendronate after denosumab.

Conclusion Based on the final results of this crossover study after women had received each treatment for up to 1 year, postmenopausal women with osteoporosis were more adherent, compliant, and persistent with subcutaneous denosumab injections every 6 months than with once-weekly alendronate tablets and reported increased treatment preference and satisfaction with injectable denosumab over oral alendronate.

Keywords Adherence - Alendronate - Compliance Denosumab $\cdot$ Persistence 


\section{Introduction}

Oral bisphosphonates are the most commonly prescribed osteoporosis medications and effectively reduce fracture risk among patients with osteoporosis. However, several large studies have reported that the majority of postmenopausal women discontinue bisphosphonate therapy within 1 year of initiation [1-3]. Persistence, compliance, and adherence to long-term bisphosphonate treatment are often inadequate, leading to suboptimal health outcomes [3-7], including increased risk of fracture [1]. Treatment adherence among patients with chronic conditions may be influenced by many factors, including patient beliefs, preferences, and satisfaction with the prescribed treatment [8-14].

Denosumab is a human monoclonal antibody with affinity and specificity for RANK ligand, thereby inhibiting osteoclast formation, function, and survival [15]. A single subcutaneous injection of denosumab, $60 \mathrm{mg}$ (Prolia ${ }^{\circledR}$ ), has been shown to increase bone mineral density (BMD) and decrease bone turnover markers for at least 6 months [16]. In clinical trials, subcutaneous denosumab once every 6 months was well tolerated, increased BMD [17-19], and significantly reduced fracture risk [20]. Denosumab was also associated with significantly greater increases in BMD at the femoral neck, trochanter, lumbar spine, and one-third radius compared with once-weekly oral alendronate treatment [19].

The Denosumab Adherence Preference Satisfaction (DAPS) study evaluated adherence (including both compliance and persistence) to 12 months of treatment with subcutaneous denosumab, $60 \mathrm{mg}$ every 6 months, and 12 months of treatment with oral alendronate, $70 \mathrm{mg}$ once weekly, using a randomized, crossover design. This enabled evaluation of the primary efficacy endpoint of adherence during the first year, as reported previously [21], as well as adherence, compliance, persistence, patient beliefs, preference, satisfaction, and bother after subjects received both treatments. In addition, the crossover design provided information about the effect of administration sequence on adherence to denosumab and alendronate. This report presents the final results from both years of the DAPS study.

\section{Methods}

Study design

Eligible subjects were randomized in a 1:1 allocation to one of two treatment sequences-denosumab/alendronate or alendronate/denosumab - and received each treatment for 1 year. All study treatments were administered open label.
One group of subjects received oral alendronate, $70 \mathrm{mg}$ once weekly, in the first year, and then crossed over to subcutaneous denosumab, $60 \mathrm{mg}$ every 6 months, in the second year (given on day 1 and month 6 of the second year). The other group received the same treatments, but in reverse order. Subjects who terminated treatment before the end of the first year of study but who agreed to therapy in the second year were allowed to cross over treatment and enter the second year early.

\section{Eligibility criteria}

This multicenter, randomized, open-label, crossover study was conducted at 20 centers in the USA and 5 centers in Canada between October 2007 and July 2010 (Appendix). Subjects enrolled were ambulatory, postmenopausal women, aged 55 years or older, with baseline BMD T-scores between -4.0 and -2.0 at the lumbar spine, total hip, or femoral neck as measured by dual energy X-ray absorptiometry. Key exclusion criteria were prior bisphosphonate or denosumab treatment, use of bone-active drugs, vitamin $\mathrm{D}$ deficiency $(<20 \mathrm{ng} / \mathrm{mL}[49.9 \mathrm{nmol} / \mathrm{L}])$, or contraindications to alendronate treatment. The study was conducted in accordance with the ethical principles of the Declaration of Helsinki. Informed consent was obtained for each subject, and an institutional review board or independent ethics committee approved the study protocol for each site.

\section{Treatment}

Study clinic personnel administered denosumab as a subcutaneous injection. Alendronate was dispensed in a bottle with a medication event monitoring system (MEMS) cap to monitor administration times and dates. Subjects were informed that the way in which they took alendronate tablets would be monitored. They were instructed to open the bottle only when taking medication and only remove one tablet at each opening. They were also instructed to follow the label dosing instructions for alendronate (ingestion on the same morning each week and avoiding lying down, eating, or drinking for at least $30 \mathrm{~min}$ after administration). All subjects received daily supplementation of calcium (1,000 mg) and vitamin D (at least $400 \mathrm{IU})$.

\section{Outcomes}

Adherence was defined as a composite of being both compliant and persistent with therapy. For denosumab, subjects were considered compliant if they received the two denosumab injections 6 months \pm 4 weeks apart; they were considered persistent if they received both injections and completed that treatment period within the study-defined time span. For alendronate, subjects were considered 
compliant if they took at least $80 \%$ of the once-weekly tablets; they were considered persistent if they took at least two tablets in the last month and completed that treatment period within the allotted time. Adherence to alendronate administration was based on MEMS data and counted a maximum of four events (i.e., consumption of four alendronate tablets) per 28-day period. The cutoff of $80 \%$ for compliance to alendronate was similar to that used in previous bisphosphonate studies [1, 2, 7]. Patients with $>80 \%$ compliance to bisphosphonate therapy have a $16 \%$ lower relative risk of fracture than patients who are less compliant [5]. Subjects who took at least two of four tablets in the last month were considered persistent to alendronate because it was assumed that some non-persistent subjects might take study treatment when they realized that the 12month follow-up visit was approaching.

At each follow-up visit, subjects completed an adaptation of the Beliefs about Medicines Questionnaire (BMQ) [22] that included 22 specific questions in the following major domains: the necessity of the prescribed medication to manage osteoporosis now and in the future (five items), concerns about the potential adverse effects of taking the prescribed medication to manage osteoporosis (ten items), and preference for one medication over the other (seven items). At each visit, subjects also completed the Preference Satisfaction Questionnaire (PSQ) [23], which measured five domains: preference, pill satisfaction, injection satisfaction, pill bother, and injection bother.

BMD (lumbar spine and hip) was assessed at baseline and the 12-month visits of each year. Fasting serum C-telopeptide (CTX-1) and N-terminal propeptide type I procollagen (P1NP) were assessed at baseline and 12 months of the first year, and 6 and 12 months after crossover.

\section{Statistical methods}

The primary endpoint was the proportion of subjects in each treatment group who were adherent to treatment at the end of the first year. Efficacy analyses used the intent-totreat principle and included all randomized subjects for the first year, and all crossover subjects for the second year. Data from both years are reported in this analysis because data that were missing at the time of the prior report [21] could be collected during the second year. Exploratory analyses of BMD and BMQ included all observed data at the time point of interest.

Safety endpoints included subject incidences of adverse events and serious adverse events. The safety population within each year of study included all subjects who received at least one dose of study medication in that year. If a subject accidentally received both study treatments in a single period, they were considered to have received denosumab for safety analyses in that period.
Statistical hypothesis tests were conducted at the 0.05 significance level. Point estimates and 95\% confidence intervals (CI) were determined for the absolute rate reduction and for the rate ratio between treatment groups for non-adherence, non-compliance, and non-persistence. These endpoints were compared between treatment groups using a Cochran-Mantel-Haenszel test stratified by center and prior osteoporotic fracture. Ordinal, categorical, patient-reported endpoints were compared between treatment groups in each treatment period using a van Elteren non-parametric test, stratified by investigational site and prior osteoporotic fracture. Treatment-by-period interactions were assessed for significance $(p$ value $<0.1)$ by statistical methods with data from both treatment periods. Time to non-adherence was defined as the time to treatment non-compliance or non-persistence, whichever occurred earliest. Non-adherence to alendronate could begin at any time. The time to denosumab non-adherence (for nonadherent subjects) was defined as 6 months and 4 weeks after the most recent injection. Time to treatment nonadherence was described with Kaplan-Meier methods without statistical comparisons.

Logistic regression analyses of non-adherence, noncompliance, and non-persistence were stratified by prior osteoporotic fracture. Potential explanatory variables explored individually in the model were baseline values (i.e., prior to study entry) for age, age group ( $<65$ or $\geq 65$ years), race (Caucasian or non-Caucasian), prior bone-loss therapy, parental hip fracture (yes or no), smoking history, alcohol intake, and time since menopause, as well as values from the start of each treatment period for total hip BMD and BMQ scores. The sample size was determined as described previously [21].

\section{Results}

Study participants

Of the 250 subjects who were originally enrolled, 221 entered the second year of treatment (106 denosumab, 115 alendronate) (Fig. 1). Baseline characteristics prior to study treatment were similar between treatment groups (Table 1).

\section{Adherence}

Adherence is summarized by study year in Table 2. Because the sequence effect (treatment-by-period interaction) was significant ( $p$ value $<0.1$ ), adherence, compliance, and persistence were reported separately for each treatment period rather than combining data from both treatment periods. 
Fig. 1 Subject disposition. Note: One subject received both study treatments in a single period and was considered to have received denosumab for safety analyses in that period. The safety population included all subjects who received at least one dose of study medication; subjects in the alendronate group were required to return at least one MEMS bottle to confirm they had received at least one dose of alendronate. Subjects were considered to have completed the period/year if the year's month 12 visit occurred within or later than the schedule visit window with "Yes" for the end-of-year completion response

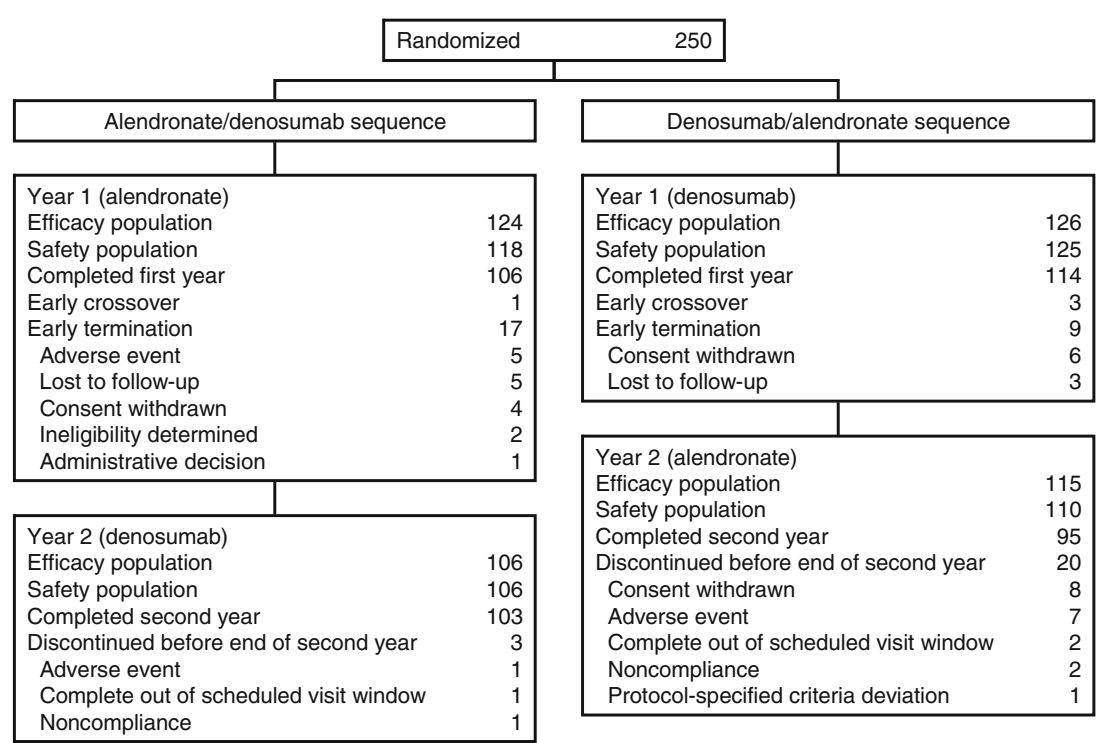

By the end of the first 12 months, $11.9 \%$ subjects were non-adherent to denosumab, and $23.4 \%$ were non-adherent to alendronate, for an absolute difference of $10.5 \%(95 \% \mathrm{CI}$ $1.3 \%, 19.7 \%$ ) adjusting for investigational site and prior osteoporosis fracture status. The rate ratio for nonadherence in the first year was 0.54 (95\% CI $0.31,0.93$; $p=0.026$ ) between treatment groups, representing a $46 \%$ reduction in the risk of non-adherence for denosumab compared with alendronate. The non-adherence rate after crossover was $7.5 \%$ for denosumab and $36.5 \%$ for alendronate, with an absolute difference of 30.9\% $(95 \%$ CI $20.6 \%, 41.3 \%$ ). The adjusted non-adherence ratio after crossover was 0.20 (95\% CI $0.10,0.41 ; p<0.001)$, representing an 80\% lower risk of non-adherence with denosumab. Time to treatment non-adherence (Fig. 2) differed early between treatments and was more pronounced after crossover.

\section{Compliance and persistence}

Results of the analyses of non-compliance and nonpersistence (Table 2) were consistent with the analyses of non-adherence for each year. Non-compliance results for the first year did not change from the previous report with the addition of new data that had been missing at the time of reporting the primary endpoint [21]. Non-compliance

Table 1 Baseline demographics and disease characteristics (efficacy populations)

\begin{tabular}{|c|c|c|c|c|}
\hline & \multicolumn{2}{|l|}{ First year of study } & \multicolumn{2}{|l|}{ Second year of study } \\
\hline & $\begin{array}{l}\text { Receiving } \\
\text { alendronate }(n=124)\end{array}$ & $\begin{array}{l}\text { Receiving } \\
\text { denosumab }(n=126)\end{array}$ & $\begin{array}{l}\text { Receiving } \\
\text { alendronate }(n=115)\end{array}$ & $\begin{array}{l}\text { Receiving } \\
\text { denosumab }(n=106)\end{array}$ \\
\hline Sex, female, $n(\%)$ & $124(100)$ & $126(100)$ & $115(100)$ & $106(100)$ \\
\hline \multicolumn{5}{|l|}{ Ethnicity/race, $n(\%)$} \\
\hline White or Caucasian & $119(96.0)$ & $115(91.3)$ & $107(93.0)$ & $102(96.2)$ \\
\hline Hispanic or Latino & $1(0.8)$ & $6(4.8)$ & $4(3.5)$ & $1(0.9)$ \\
\hline Black or African American & $2(1.6)$ & $2(1.6)$ & $1(0.9)$ & $1(0.9)$ \\
\hline Other & $2(1.6)$ & $3(2.4)$ & $3(2.6)$ & $2(1.9)$ \\
\hline Age, years, mean (SD) & $65.3(7.7)$ & $65.1(7.6)$ & $65.1(7.4)$ & $65.3(7.4)$ \\
\hline Years since menopause, mean (SD) & $17.2(10.0)$ & $18.2(11.4)$ & $17.9(10.9)$ & $17.0(9.7)$ \\
\hline \multicolumn{5}{|c|}{ BMD T-scores at year baseline, mean (SD) } \\
\hline Lumbar spine & $-1.89(1.13)$ & $-2.04(1.16)$ & $-1.61(1.29)$ & $-1.44(1.15)$ \\
\hline Total hip & $-1.60(0.76)$ & $-1.60(0.74)$ & $-1.38(0.74)$ & $-1.40(0.73)$ \\
\hline Femoral neck & $-2.03(0.62)$ & $-2.01(0.55)$ & $-1.84(0.60)$ & $-1.90(0.63)$ \\
\hline
\end{tabular}

Values are given for baseline (start of the first year)

$S D$ standard deviation, $B M D$ bone mineral density 
Table 2 Subject non-adherence, non-compliance, and non-persistence (efficacy populations)

\begin{tabular}{|c|c|c|c|c|c|}
\hline & \multicolumn{2}{|c|}{ Crude rate, $n(\%)$} & \multirow{2}{*}{$\begin{array}{l}\text { Absolute rate }{ }^{a} \text { reduction } \\
(95 \% \mathrm{CI})\end{array}$} & \multirow{2}{*}{$\begin{array}{l}\text { Rate ratio }{ }^{a} \\
(95 \% \mathrm{CI})\end{array}$} & \multirow[t]{2}{*}{$p$ value $^{\mathrm{a}}$} \\
\hline & Denosumab & Alendronate & & & \\
\hline First year & $(n=126)$ & $(n=124)$ & & & \\
\hline Adherence $^{\mathrm{b}}$ & $111(88.1)$ & $95(76.6)$ & & & \\
\hline Non-adherence & 15 (11.9) & $29(23.4)$ & $10.5(1.3,19.7)$ & $0.54(0.31,0.93)$ & 0.026 \\
\hline Compliance $^{c}$ & $114(90.5)$ & $97(78.2)$ & & & \\
\hline Non-compliance & $12(9.5)$ & $27(21.8)$ & $11.0(2.2,19.7)$ & $0.48(0.26,0.87)$ & 0.014 \\
\hline Persistence $^{\mathrm{d}}$ & $114(90.5)$ & $99(79.8)$ & & & \\
\hline Non-persistence & $12(9.5)$ & $25(20.2)$ & $9.8(1.1,18.5)$ & $0.50(0.27,0.93)$ & 0.029 \\
\hline Second year & $(n=106)$ & $(n=115)$ & & & \\
\hline Adherence $^{\mathrm{b}}$ & $98(92.5)$ & $73(63.5)$ & & & \\
\hline Non-adherence & $8(7.5)$ & $42(36.5)$ & $30.9(20.6,41.3)$ & $0.20(0.10,0.41)$ & $<0.001$ \\
\hline Compliance $^{c}$ & $99(93.4)$ & $78(67.8)$ & & & \\
\hline Non-compliance & $7(6.6)$ & $37(32.2)$ & $27.7(17.6,37.7)$ & $0.20(0.09,0.43)$ & $<0.001$ \\
\hline Persistence $^{\mathrm{d}}$ & $103(97.2)$ & $82(71.3)$ & & & \\
\hline Non-persistence & $3(2.8)$ & $33(28.7)$ & $27.4(18.1,36.7)$ & $0.09(0.03,0.30)$ & $<0.001$ \\
\hline
\end{tabular}

${ }^{a}$ Based on the Cochran-Mantel-Haenszel method stratified by center and prior osteoporotic fracture

${ }^{\mathrm{b}}$ Adherence was defined as satisfying the criteria for both compliance and persistence

${ }^{\mathrm{c}}$ Compliance was defined as receiving two injections 6 months \pm 4 weeks apart (denosumab) or at least $80 \%$ of weekly doses (alendronate)

${ }^{\mathrm{d}}$ Persistence was defined as receiving either two injections total (denosumab) or at least two weekly doses in the last month (alendronate), and completing the year of treatment within the allotted time (both groups)

after crossover was $6.6 \%$ for denosumab and $32.2 \%$ for alendronate, with an absolute difference of $27.7 \%$ (95\% CI $17.6 \%, 37.7 \%)$; the adjusted rate ratio was 0.20 (95\% CI $0.09,0.43 ; p<0.001)$, representing an $80 \%$ relative risk reduction of non-compliance with denosumab.

Non-persistence in the first year was $9.5 \%$ for denosumab and $20.2 \%$ for alendronate, with an absolute difference of $9.8 \%(95 \%$ CI $1.1 \%, 18.5 \%)$; the adjusted rate ratio was 0.50 (95\% CI 0.27, 0.93; $p=0.029)$, representing a $50 \%$ relative risk reduction of non-persistence with denosumab. Non-persistence after crossover was $2.8 \%$ for denosumab and $28.7 \%$ for alendronate, with an absolute difference of $27.4 \%(95 \%$ CI $18.1 \%, 36.7 \%)$; the adjusted rate ratio was 0.09 (95\% CI $0.03,0.30 ; p<0.001)$, representing a $91 \%$ relative risk reduction of non-persistence with denosumab.

\section{Patient-reported outcomes}

Figure 3 summarizes BMQ scores at each study visit. Mean scores for subject beliefs about the necessity for the prescribed treatment were greater for denosumab than for alendronate at the 6-month visit in the first year $(p=0.022)$,
Fig. 2 Time to treatment nonadherence. Non-adherence to alendronate could begin at any time, and the time to non-adherence was defined as the time to treatment noncompliance or time to treatment non-persistence, whichever occurred earliest. The time to denosumab non-adherence for non-adherent subjects was defined as 6 months and 4 weeks after the most recent injection. For each treatment group, time points with $>95 \%$ cumulated subjects were excluded
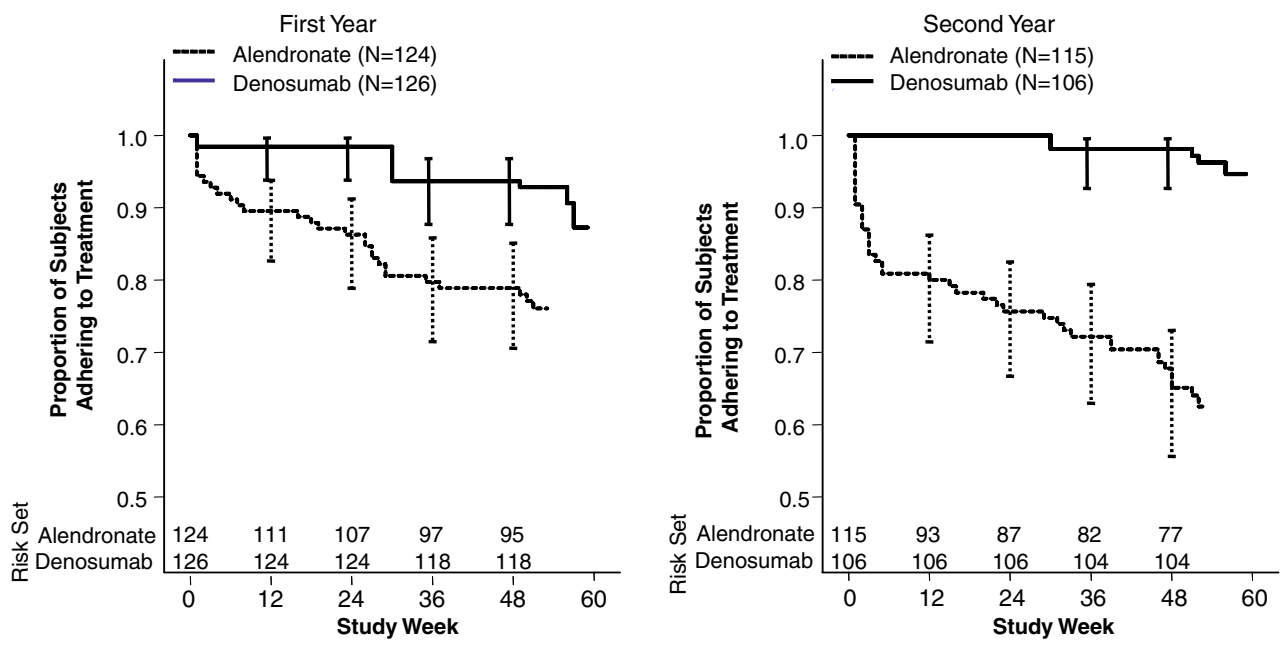
Fig. 3 Mean scores on the BMQ. ${ }^{*} p<0.05$ between treatment groups. ${ }^{\dagger} p<0.05$ between treatment groups for difference in change score from each year's baseline. ${ }^{\ddagger} n$ values are shown for the number of subjects with observed data in the first and second years, respectively; the latter population was used for the analysis of scores at the crossover visit. ${ }^{\S}$ Visit 1 baseline; visit 2 year 1 , month 6 ; visit 3 crossover (BMQ baseline of year 2 treatment); visit 4 year 2 , month 6 ; visit 5 year 2 , month 12 . Total score ranged from 1 to 5 . Higher scores indicate stronger beliefs, concerns, and preference

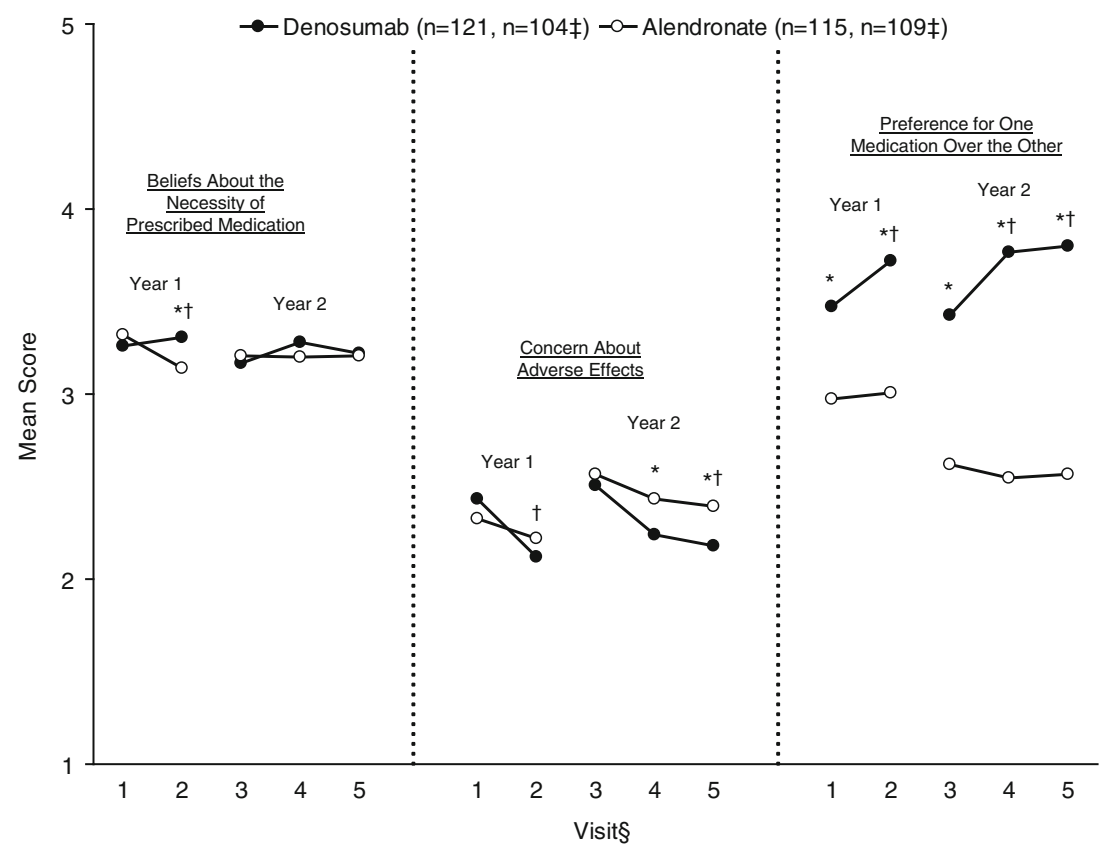

but not at the other visits. Mean scores for subject concerns about potential adverse consequences of treatment were lower for denosumab than for alendronate at the 6month $(p=0.010)$ and 12-month $(p=0.028)$ visits after crossover, but not at the other time points. Mean scores for subject preference for one medication over the other were greater for denosumab than for alendronate at every visit (all $p<0.001$ ).

At the end of study, of the 198 subjects who expressed a preference between treatments, 183 (92.4\%) preferred subcutaneous denosumab injections over alendronate tablets $(p<0.001)$ (Online resource 1). Of the 204 subjects who expressed a preference between treatments for the long term, $186(91.2 \%)$ said they would choose denosumab injections for long-term treatment $(p<0.001)$ (Online resource 1).

Figure 4 summarizes PSQ subject satisfaction scores at the end of each treatment period. Regardless of the treatment sequence, a greater proportion of subjects reported they were quite/very satisfied with frequency of administration, mode of administration, and convenience of denosumab compared with alendronate.

Logistic regression analyses of non-adherence

Analyses of non-adherence based on multiple logistic regressions indicated that the only significant subject characteristic in the first year was total hip BMD $(p=0.028)$ (Online resource 2). Significant subject characteristics after crossover were BMQ scores for necessity $(p=0.006)$, concern $(p=$ $0.025)$, and preference $(p=0.024)$.
Exploratory endpoints: bone mineral density and bone turnover markers

Mean percentage changes in BMD (observed data) in the first year for the alendronate and denosumab groups, respectively, were as follows: lumbar spine, $4.9 \%(n=93)$ and 5.6\% $(n=93)$; total hip, $2.5 \%(n=102)$ and $3.2 \%(n=$ $109)$; and femoral neck, $2.0 \%(n=102)$ and $3.1 \%(n=109)$. Mean percentage BMD changes from baseline of the second year to the end of treatment for alendronate and denosumab, respectively, were as follows: lumbar spine, $0.6 \%(n=82)$ and $2.9 \%(n=92)$; total hip, $0.4 \%(n=92)$ and $1.5 \%(n=102)$; and femoral neck, $-0.1 \%(n=92)$ and $1.7 \%$ $(n=102)$.

Median CTX-1 levels at baseline, the end of the first year, and the end of treatment, respectively, were as follows: denosumab/alendronate sequence, $0.465 \mathrm{ng} / \mathrm{mL}$ $(n=75), 0.139 \mathrm{ng} / \mathrm{mL}(n=108)$, and $0.223 \mathrm{ng} / \mathrm{mL}(n=92)$; alendronate/denosumab sequence, $0.435 \mathrm{ng} / \mathrm{mL} \quad(n=81)$, $0.132 \mathrm{ng} / \mathrm{mL}(n=100)$, and $0.140 \mathrm{ng} / \mathrm{mL}(n=100)$. Median values for P1NP levels at baseline, the end of the first year, and the end of treatment, respectively, were as follows: denosumab/alendronate sequence, $50.06 \mu \mathrm{g} / \mathrm{L} \quad(n=75)$, $14.97 \mu \mathrm{g} / \mathrm{L}(n=108)$, and $21.73 \mu \mathrm{g} / \mathrm{L}(n=92)$; alendronate/ denosumab sequence, $53.37 \mu \mathrm{g} / \mathrm{L} \quad(n=81), 17.26 \mu \mathrm{g} / \mathrm{L}$ $(n=100)$, and $16.96 \mu \mathrm{g} / \mathrm{L}(n=100)$.

At baseline, no subject in either treatment group had a CTX-1 level below the limit of quantification. At the end of the first year, $2 / 108(1.9 \%)$ subjects in the denosumab group and $3 / 100(3.0 \%)$ subjects in the alendronate group 
Fig. 4 Subject-reported satisfaction with alendronate or denosumab at the end of the study. *Alendronate/denosumab group $(A L N / D M A B)$ : data were from the last measurements of the first year for alendronate and the last measurements of the second year for denosumab. ${ }^{\dagger}$ Denosumab/alendronate group $(D M A B / A L N)$ : data were from the last measurements of the first year for denosumab and the last measurements of the second year for alendronate. ${ }^{\ddagger}$ Combined: includes combined data from both groups

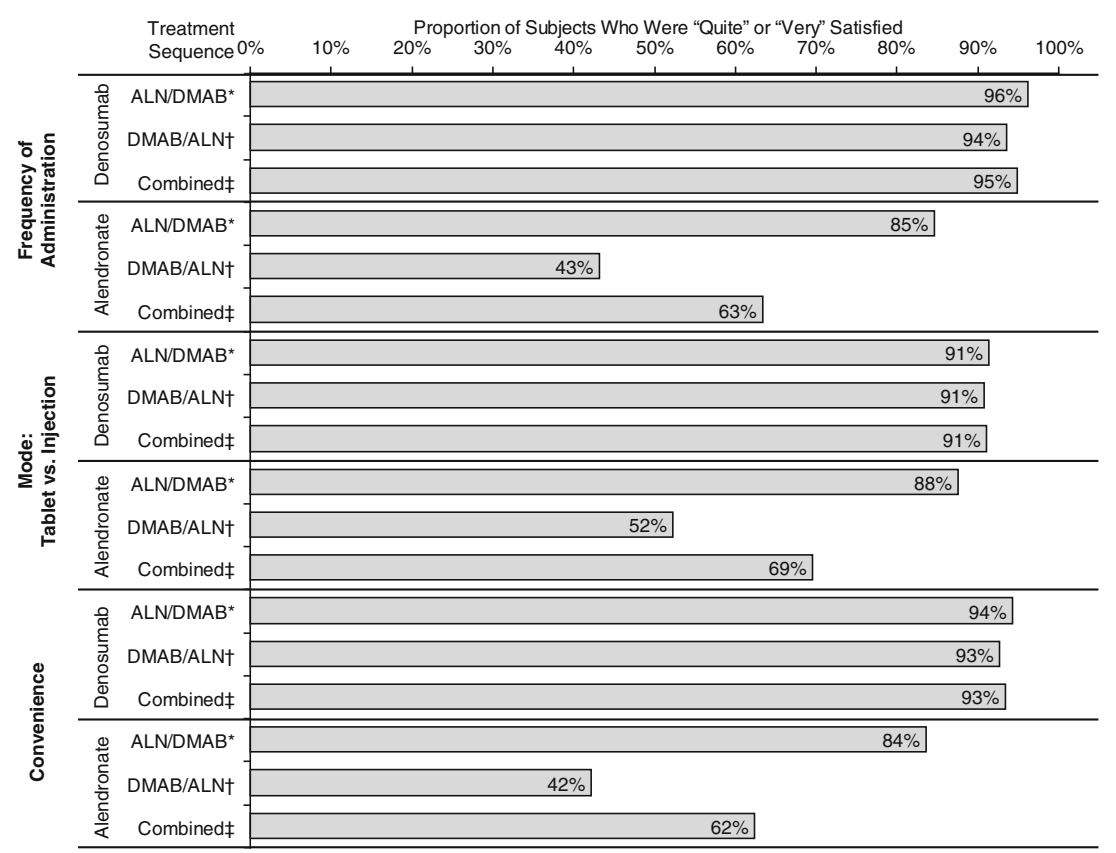

had undetectable CTX-1 levels. Six months after crossover, $13 / 86(15.1 \%)$ subjects in the denosumab group and $4 / 97$ (4.1\%) subjects in the alendronate group had undetectable CTX-1 levels. At the end of study, 15/100 (15.0\%) subjects in the denosumab group and 6/92 (6.5\%) subjects in the alendronate group had undetectable CTX-1 levels.

\section{Safety}

The safety population included 228 subjects who received at least one dose of alendronate and 230 subjects who received at least one dose of denosumab. Adverse events with incidence rates $>2 \%$ by preferred term in either treatment group were not significantly different between treatment groups in the second treatment period. Overall, $63.2 \%$ and $65.7 \%$ of subjects reported at least one adverse event during alendronate and denosumab treatment, respectively. Adverse events reported by at least $5 \%$ of subjects during either treatment (alendronate, denosumab) were arthralgia $(6.6 \%, 6.1 \%)$, pain in extremity $(3.9 \%, 6.1 \%)$, and back pain $(5.7 \%, 3.9 \%)$.

Adverse events of fracture during the first year included one subject with fibula fracture during alendronate treatment and one with foot fracture during denosumab treatment. Adverse events of fracture after crossover included one subject with humerus fracture during alendronate treatment and one subject each with foot, pubis, and ulna fracture during denosumab treatment.

Serious adverse events occurred in $3.9 \%$ and $3.5 \%$ of subjects during alendronate and denosumab treatment, respectively. The only serious adverse event in more than one subject was osteoarthritis, which was reported for three
$(1.3 \%)$ subjects during denosumab treatment. None of the serious adverse events was considered related to study treatment. No deaths, osteonecrosis of the jaw, or atypical femoral fractures were reported.

\section{Discussion}

In this study, postmenopausal women who received subcutaneous injections of denosumab every 6 months had significantly better adherence, compliance, and persistence than women who self-administered alendronate orally once weekly. Non-adherence and non-persistence in the first year favored denosumab slightly more in the present analysis than in the prior report [21] because one subject had missing information at the time of the prior analysis.

Non-adherence, non-compliance, and non-persistence rates for alendronate-treated subjects were higher after crossover from denosumab; the rates were lower for denosumab-treated subjects after crossover from alendronate. These observations suggest there may be a treatment sequence effect: transitioning from biannual to weekly administration may have been more difficult to follow than the converse, an observation that has been noted elsewhere [24].

The BMQ survey results provided insights into subjects' impressions of denosumab and alendronate. In each treatment year, subjects felt the therapy was necessary for their osteoporosis, regardless of mode of administration. Even though subjects believed in the necessity of treatment, they were not fully adherent to either treatment, although more so with alendronate. Subjects were significantly less 
concerned about the potential for adverse consequences with denosumab administration than with alendronate administration, but only after crossover, when they had experienced both forms of treatment administration. Of the subjects who expressed a preference for either therapy at the end of study, more than $90 \%$ said they preferred the injectable therapy over the tablets, and they would prefer the injections for long-term treatment. Subject belief and preference scores at each visit also tended to favor denosumab, and they generally improved more during denosumab treatment than during alendronate treatment.

The administration route for denosumab is likely to influence patient adherence to treatment. The injectable formulation of denosumab requires subcutaneous administration by healthcare professionals, giving them a greater role in ensuring patients adhere to treatment. It also provides healthcare professionals with direct evidence of patient adherence to treatment - and an opportunity to communicate with the patient about the importance of not missing scheduled treatments - rather than relying on patient recall about their treatment adherence.

This study used an open-label, crossover design to assess adherence and patient-reported outcomes. Two double-blind studies involving denosumab and alendronate had previously analyzed patient preference and satisfaction data [11]; however, queries were restricted to the mode of administration (injection vs tablet) and dosing frequency (once weekly vs every 6 months) because subjects were not informed of their treatment assignment. In the current evaluation, it was important for subjects to know what treatment they had received to evaluate their overall satisfaction with treatment in a situation that mimicked routine clinical practice to the extent possible.

Follow-up visits with bisphosphonate treatment typically occur annually; however, visits in this study occurred every 6 months, which could have enhanced adherence. Additionally, adherence to weekly alendronate treatment required subjects to take at least $80 \%$ of the tablets, including two of four doses $(50 \%)$ in the final month; in contrast, denosumab adherence required administration of $100 \%$ of the doses, possibly biasing against denosumab for adherence. If adherence to alendronate treatment in this study had required administration of $100 \%$ of doses with four doses in the final month, the rates of alendronate adherence would have been substantially lower $(18.5 \%$ in the first year and $11.3 \%$ after crossover).

Study definitions for adherence were selected to focus on intake of study medication and not clinical benefit to the subject. Oral alendronate treatment is approved for clinical use in once-daily and once-weekly regimens, based on evidence of the clinical benefits of these dosing regimens. Despite evidence that alendronate remains in the bone matrix for many years and is gradually released as bone is resorbed [25], the magnitude and duration of this effect is uncertain. It has been reported that stopping alendronate treatment after 4 to 5 years results in a significant increase in clinical vertebral fractures, but a residual clinical efficacy for nonvertebral fractures [26]. It has also been reported that patients who had discontinued bisphosphonate treatment in the previous 6 months had similar fracture risks as patients who discontinued more distantly and as patients who just started treatment, suggesting there is little residual effect on fracture risk reduction after stopping bisphosphonates [27]. Thus, although it is possible that subjects who were non-persistent after receiving alendronate for at least 6 months experienced a carry-over effect for the subsequent 6 months, these effects may not necessarily translate to clinical benefits for the subject.

Conceptual models have documented the relationship between non-adherence and cost-effectiveness or value to the healthcare system $[4,28]$. An economic model of the DAPS results that were reported after the first year concluded, "Denosumab is a cost-effective alternative to oral osteoporosis treatments, particularly for patients at high risk of fracture and low expected adherence to oral treatments" [29].

The rate of alendronate non-adherence in this study ( $23 \%$ in the first year) was lower than in other retrospective observational reports (33\% to $50 \%$ in the first year) that also used the $80 \%$ threshold for alendronate adherence [1, $2,7]$. One possible reason for this difference was that subjects in this study knew that their adherence was being monitored. Additionally, they knew they would switch treatment at the crossover, and their BMD was being monitored, each of which may enhance bisphosphonate treatment adherence [2]. Other observational studies have reported even higher rates of bisphosphonate nonadherence $(50 \%$ to $80 \%)$ with longer follow-up (1.7 to 2.0 years) $[2,3,5,6]$. Thus, the use of 1 -year treatment periods in this study limits the conclusions that can be made about long-term compliance with either treatment. Another potential study limitation was that the study sponsor provided alendronate and denosumab to the subjects, which removed any influence of treatment cost on adherence. The study was conducted at centers in North America (USA and Canada), and caution is warranted in the extrapolation of these results in other regions.

Consistent with other denosumab studies [18, 19], both treatments were well tolerated, and adverse events were similar between groups in this study. Also consistent with those prior studies, exploratory analyses from this study indicated that subjects who crossed over from alendronate to denosumab continued to have increases in BMD and reduction of bone turnover markers in the second year. Subjects who transitioned from denosumab to alendronate treatment had BMD that remained stabilized from the increases observed while on denosumab and bone turnover 
marker levels that increased slightly. This is the first report showing BMD and bone turnover marker levels for subjects transitioning from denosumab to alendronate.

In summary, this study showed that postmenopausal women with low BMD who received alendronate followed by denosumab, or denosumab followed by alendronate, preferred treatment with subcutaneous injections of denosumab every 6 months. Increased preference may influence persistence and adherence with therapy, important characteristics in treatment of a chronic condition that requires long-term treatment.

Acknowledgments The DAPS study was sponsored by Amgen Inc. and is registered in ClinicalTrials.gov under the identifier NCT00518531. Jonathan Latham and Yeshi Mikyas provided medical writing assistance on behalf of Amgen Inc. Christine Fletcher of Amgen Inc. provided extensive support with the study design and statistical analysis plan.

Conflicts of interest N. Freemantle has received research grants from Amgen and has served as a consultant for Amgen, SanofiAventis, Pfizer, Wyeth, and Eli Lilly. S. Satram-Hoang has served as a consultant for Amgen. E. Tang, P. Kaur, D. Macarios, and S. Siddhanti are employees and shareholders of Amgen. J. Borenstein previously was employed by Amgen. D. Kendler has received grant or research support from Amgen, Merck, Eli Lilly, Novartis, Procter \& Gamble, GlaxoSmithKline, Pfizer, Roche Biosante, and Wyeth and has served as an advisor for Amgen, Merck, Eli Lilly, Novartis, Wyeth, Nycomed, Procter \& Gamble, and Pfizer.

Open Access This article is distributed under the terms of the Creative Commons Attribution Noncommercial License which permits any noncommercial use, distribution, and reproduction in any medium, provided the original author(s) and source are credited.

\section{Appendix}

The Denosumab Adherence Preference Satisfaction (DAPS) study investigators were as follows, listed alphabetically by country: USA-Bruce Akright, Kurt Datz, Ara Dikranian, Elyse Erlich, Stephen Fehnel, Catherine Gerrish, John Joseph, Robert Lang, Leroy Leeds, Michael Lillestol, Dennis Linden, Michael McClung, Jefferey Michelson, Alfred Moffett, Constantine Saadeh, Gerald Shockey, Joseph Soufer, Raul Tamayo, and John Williams; Canada-Jonathan Adachi, Stephanie Kaiser, David Kendler, Jean-Pierre Raynauld, and Jerieta Waltin-James.

\section{References}

1. Imaz I, Zegarra P, González-Enríquez J, Rubio B, Alcazar R, Amate JM (2010) Poor bisphosphonate adherence for treatment of osteoporosis increases fracture risk: systematic review and metaanalysis. Osteoporos Int 21:1943-1951
2. Kothawala P, Badamgarav E, Ryu S, Miller RM, Halbert RJ (2007) Systematic review and meta-analysis of real-world adherence to drug therapy for osteoporosis. Mayo Clin Proc 82:1493-1501

3. Siris ES, Harris ST, Rosen CJ, Barr CE, Arvesen JN, Abbott TA, Silverman S (2006) Adherence to bisphosphonate therapy and fracture rates in osteoporotic women: relationship to vertebral and nonvertebral fractures from 2 US claims databases. Mayo Clin Proc 81:1013-1022

4. Hiligsmann M, Rabenda V, Gathon HJ, Ethgen O, Reginster JY (2010) Potential clinical and economic impact of nonadherence with osteoporosis medications. Calcif Tissue Int $86: 202-210$

5. Caro JJ, Ishak KJ, Huybrechts KF, Raggio G, Naujoks C (2004) The impact of compliance with osteoporosis therapy on fracture rates in actual practice. Osteoporos Int 15:1003-1008

6. Huybrechts KF, Ishak KJ, Caro JJ (2006) Assessment of compliance with osteoporosis treatment and its consequences in a managed care population. Bone 38:922-928

7. Yeaw J, Benner JS, Walt JG, Sian S, Smith DB (2009) Comparing adherence and persistence across 6 chronic medication classes. J Manag Care Pharm 15:728-740

8. Brown C, Battista DR, Bruehlman R, Sereika SS, Thase ME, Dunbar-Jacob J (2005) Beliefs about antidepressant medications in primary care patients: relationship to self-reported adherence. Med Care 43:1203-1207

9. Byer B, Myers LB (2000) Psychological correlates of adherence to medication in asthma. Psychol Health Med 5:389-393

10. Horne R, Buick D, Fisher M, Leake H, Cooper V, Weinman J (2004) Doubts about necessity and concerns about adverse effects: identifying the types of beliefs that are associated with nonadherence to HAART. Int J STD AIDS 15:38-44

11. Kendler DL, Bessette L, Hill CD et al (2010) Preference and satisfaction with a 6-month subcutaneous injection versus a weekly tablet for treatment of low bone mass. Osteoporos Int 21:837-846

12. Fallowfield L, Atkins L, Catt S et al (2006) Patients' preference for administration of endocrine treatments by injection or tablets: results from a study of women with breast cancer. Ann Oncol 17:205-210

13. Granger AL, Fehnel SE, Hogue SL, Bennett L, Edin HM (2006) An assessment of patient preference and adherence to treatment with Wellbutrin SR: a web-based survey. J Affect Disord 90:217221

14. Reginster JY, Rabenda V, Neuprez A (2006) Adherence, patient preference and dosing frequency: understanding the relationship. Bone 38:S2-S6

15. Kostenuik PJ (2005) Osteoprotegerin and RANKL regulate bone resorption, density, geometry and strength. Curr Opin Pharmacol 5:618-625

16. Bekker PJ, Holloway DL, Rasmussen AS et al (2004) A singledose placebo-controlled study of AMG 162, a fully human monoclonal antibody to RANKL, in postmenopausal women. J Bone Miner Res 19:1059-1066

17. McClung MR, Lewiecki EM, Cohen SB et al (2006) Denosumab in postmenopausal women with low bone mineral density. N Engl J Med 354:821-831

18. Lewiecki EM, Miller PD, McClung MR et al (2007) Two-year treatment with denosumab (AMG 162) in a randomized phase 2 study of postmenopausal women with low BMD. J Bone Miner Res 22:1832-1841

19. Brown JP, Prince RL, Deal C et al (2009) Comparison of the effect of denosumab and alendronate on BMD and biochemical markers of bone turnover in postmenopausal women with low bone mass: a randomized, blinded, phase 3 trial. J Bone Miner Res 24:153161 
20. Cummings SR, San Martin J, McClung MR et al (2009) Denosumab for prevention of fractures in postmenopausal women with osteoporosis. N Engl J Med 361:756-765

21. Kendler DL, McClung MR, Freemantle N et al (2011) Adherence, preference, and satisfaction of postmenopausal women taking denosumab or alendronate. Osteoporos Int 22:1725-1735

22. Horne R, Weinman J, Hankins M (1999) The beliefs about medicines questionnaire: the development and evaluation of a new method for assessing the cognitive representation of medication. Psychol Health 14:1-24

23. Gold DT, Horne R, Hill C, Borenstein J, Varon S, Macarios D (2008) Development, reliability, and validity of a new preference satisfaction questionnaire (PSQ). J Bone Miner Res 23:S210-S211

24. Briesacher BA, Andrade SE, Harrold LR, Fouayzi H, Yood RA (2010) Adherence and occurrence of fractures after switching to once-monthly oral bisphophonates. Pharmacoepidemiol Drug Saf 19:1233-1240
25. Rodan G, Reszka A, Golub E, Rizzoli R (2004) Bone safety of long-term bisphosphonate treatment. Curr Med Res Opin 20:1291-1300

26. Black DM, Schwartz AV, Ensrud KE et al (2006) Effects of continuing or stopping alendronate after 5 years of treatment: the Fracture Intervention Trial Long-term Extension (FLEX): a randomized trial. JAMA 296:2927-2938

27. Gallagher AM, Rietbrock S, Olson M, van Staa TP (2008) Fracture outcomes related to persistence and compliance with oral bisphosphonates. J Bone Miner Res 23:1569-1575

28. Ström O, Borgström F, Kanis JA, Jönsson B (2009) Incorporating adherence into health economic modelling of osteoporosis. Osteoporos Int 20:23-34

29. Jönsson B, Ström O, Eisman JA et al (2011) Cost-effectiveness of denosumab for the treatment of postmenopausal osteoporosis. Osteoporos Int 22:967-982 